\title{
Cross-functional team as a high-performance practice for sustainable development of Russian companies
}

\author{
Elena Kalabina*, and Olga Belyak \\ Ural State University of Economics, 8 Marta st., 62, 620144 Yekaterinburg, Russia
}

\begin{abstract}
The article considers cross-functional teams of employees as a highly productive work practice to ensure the sustainable development of companies. Dissemination of the best high-performance work practices and social work with personnel, including designing cross - functional teams, is one of the areas that ensure the sustainable development of companies. However, the design of cross-functional teams within organizations faces various difficulties and, mainly, with fragmentation and mismatch of ideas about this type of group work and methods of managing it. Rationale of expediency of applying the practice of forming cross-functional teams of employees is given. A contextual analysis of the reasons for creating crossfunctional teams of employees is carried out, their polyphonic nature is revealed. The study examines the signs of the exclusivity of crossfunctional teams of employees as an organizational unit, project team, organizational knowledge generator, cross-cultural phenomenon and presents the results of a pilot study of organizational and managerial factors of the impact of knowledge exchange between participants of crossfunctional teams to ensure the sustainable development of companies.
\end{abstract}

\section{Introduction}

It is known that the concept of sustainable development began in the late 1960s from the opinions of scientists warning humanity that the growth of the consumption economy, which then prevailed in developed countries, would soon lead to the depletion of the planet's resources.

Since then, the concept has become more systematic, the problems that humanity faces on the way to sustainable development are not limited exclusively to environmental ones, the circle has expanded due to the social dimension, the level of understanding has significantly increased.

The UN has developed an extensive tree of sustainable development goals (including 17 different purposes) and practical tools for adjusting the socio-economic model to achieve it.

One of the five key (sometimes conflicting) areas of sustainable development of companies, along with the economy, the cooperation with small and medium-sized

\footnotetext{
* Corresponding author: kalabina@mail.ru
} 
businesses, the environment, the development of the region of presence, is social policy and personnel management. These goals are multifaceted and sometimes contradictory: for example, the economy of companies, which implies its financial sustainability, faces restrictions related to the lack of staff turnover, and the environment - with the level of additional costs for the use of the best expensive technologies that reduce emissions.

For leading participants of Russian markets, sustainable development is not following fashionable civilizational trends or global trends, but rather a conscious and rational choice and strategic behavior, which allows us to consider the social policy of companies as an attempt to maintain the achieved balance of forces between employers and employees, government and business.

The social policy of personnel management develops to a greater extent according to the natural laws of the business itself and its scale is determined by the action of a whole group of factors, the main of which are: innovative activity, the size of the company, profitability and market conditions, the level of competition in the labor market, the need to retain and develop staff.

The idea of identifying a set of specific methods of personnel management that ensure high organizational results of companies, and, in particular, for sustainable growth, and known as High Performance Work Practices (HPWP), was proposed by D. Pfeffer.

The value of these practices for the sustainable development of companies is related to the fact that they can have two mutually reinforcing consequences: on the one hand, lead to improved organizational results, and on the other, contribute to improving the quality of working life, the success of social policies of companies and increasing employee satisfaction.

The origin of the term "High Performance Work Practices" is associated with the debate about what characterizes the successful management of people and company social policy, initiated by the US Department of Labor [1]. The term has gained popularity in the scientific literature of human resource management, but it should be noted that the number and composition of these practices is constantly expanding and supplementing: initially it included the so-called "magnificent seven" by J. Pfeffer [2], then it was transformed into the thirteen practices of M. Huselid [3] and the list is constantly changing. However, by now we can talk about achieving a certain "status quo": when it comes to HPWP, we mean a set of complementary and mutually reinforcing tools for personnel management, which are divided into three groups, each of which has its own purpose in terms of influencing the results of the company's activities: these are methods of ensuring a high level of employee involvement in their work and in the functioning of the organization as a whole, traditional methods of personnel management, as a rule, related to the main sub-functions of the human resource management system, and methods for ensuring high commitment of employees to the organization.

Among the newly emerging high performance work practices, it is worth paying attention to the creation of cross-functional teams in organizations.

\section{Resources and research methods}

A cross-functional team can serve as a good springboard for overcoming structural and functional fragmentation in a company that hinders effective business interaction of its employees [4]. Also, the use of cross-functional teams in the company as a modern work practice allows not only to increase the mobility of functional divisions [5], but also to get a synergistic effect from the joint group activities of employees of different areas when working on a common task or project [6]. 
Turning to cross-functional teams in organizing the work of employees, it is important for company managers to understand the features that are inherent in this form of employee interaction, which will allow choosing the most effective mechanisms for managing them.

The very name of cross-functional teams explains their main difference from other types of team interaction: when working on a project, cross-functional teams "cross" adjacent functional areas. This feature can serve in many ways as an advantage (for example, reducing errors in the development of solutions due to a comprehensive study of issues), but it is also a cornerstone (trouble) when it is necessary to create and develop a sense of community in the team, establish a highly effective knowledge exchange, and therefore effective collaboration [7].

By looking at cross-functional teams from different sides, you can see the signs of exclusivity that will allow you to fully understand the possibilities and limitations of their use, as well as methods and management mechanisms.

First, cross-functional teams are project teams, which means that they follow the standard methods and mechanisms of project management, go through the standard stages of formation, becoming, dissolution and submit to one project leader (manager). The work of the project manager in terms of team management is aimed at creating effective interaction between participants and the exchange of knowledge.

Second, a cross-functional team can be considered as an organizational unit with a clear structure, hierarchy of subordination, and degree of responsibility. In terms of team building, the greatest importance is given to the soft competencies of potential participants, for example, the ability to work in a team, flexibility of thinking and openness to new ideas. By presenting the cross-functional team by the basic components of the organizational structure, you can see that it is built according to the principles of an adaptive project structure, but at the same time it is embedded in the existing construct of the company, which uses a matrix type of organizational structure. Thus, a cross-functional team is a complex, adaptive, dynamic system that, being a part of a company, is organically integrated into its contexts and fulfills the innovative task assigned to it in the form of a project with clear resource boundaries (time, financial, organizational, labor).

Third, cross-functional teams can serve as the basis of a company's knowledge management system, since the creation and use of these teams has an innovative focus, which means it serves to accept and disseminate new knowledge in the company. The basis of this process is the effective exchange of knowledge between the participants. On the other hand, empirical research on the development of a company's absorption capacity shows that cross-functional teams can have a mixed deterrent effect on the development of absorption capacity, and it is not easy to convert potential absorption capacity into realized absorption capacity [8]. The reasons of this phenomenon are not sufficiently analyzed, however, we can assume the negative impact of insufficiently developed communication practice.

Finally, a cross-functional team is a cross-cultural phenomenon that unites people with different professional cultures, vocabulary, and personal goals. Creating an atmosphere of acceptance for all participants and developing internal communication practices in order to establish knowledge exchange is a priority for team and company managers. That is, the three dimensions of cross-functional cooperation (joint orientation to perform tasks, joint communication and joint interpersonal relations) directly determine the effective behavior in the exchange of knowledge in the cross-functional team [9]. Summarizing the above, we note that the effectiveness of a cross-functional team is based on the exchange of knowledge and best communication practices of participants, overcoming cultural professional barriers and creating a sense of community in the team.

Based on this, the purpose of our study was formed, which consists in assessing the impact of organizational and managerial factors on the exchange of knowledge in cross- 
functional teams in modern Russian conditions. To achieve this goal, a number of hypotheses have been put forward regarding the existence and nature of the relationship between various coordination mechanisms (management factors), organizational environment and organizational conditions (organizational factors) and individual activity in knowledge exchange, i.e. the degree of individual participation in knowledge exchange. Additionally, the influence of internal and external motivation for knowledge exchange on the degree of individual participation in knowledge exchange of employees involved in cross-functional teams is analyzed.

In preparation for the pilot study, a representative expert group of representatives of companies of the Ural region who participate or have experience in cross-functional teams in the course of their work was selected. The pool of companies whose representatives participated in the study included both enterprises from different industrial sectors (mining, mining processing, construction, instrument making), as well as organizations from the field of consulting and social services.

The research was carried out using a questionnaire constructed on the basis of a 6-point Likert scale, tunnel and sectional approaches. Some of the questions for the questionnaire are borrowed from foreign scientific sources with cross-cultural adaptation. The questionnaire covers organizational and managerial factors acting in the team, as well as the motives for sharing knowledge. The main dependent variable is the individual activity in the exchange of knowledge - the degree of individual participation in the exchange of knowledge.

The questionnaire consisted of four blocks that reflect micro - and macro-level factors that affect the exchange of knowledge in cross-functional teams. The macro-level factors include the coordination mechanisms acting in the team, the role of the leader (team and functional), the organizational environment and organizational conditions that facilitate the exchange of knowledge. As micro-level factors, the questionnaire takes into account individual characteristics of cross-functional team members, such as motivations for knowledge exchange, individual attitudes to knowledge exchange of team members, which directly affect the individual behavior of participants in the transfer and acceptance of knowledge from colleagues. A separate block contains questions regarding the demographic characteristics of the respondents.

Standard software tools for statistical data processing were used to analyze the results obtained.

\section{Research results}

The study included 31 respondents with experience of collaboration in cross-functional teams, including 10 women and 21 men.

All respondents have completed higher education. Distribution of the surveyed group by the type of problem to be solved, people: product - $16(52 \%)$, process $-5(16 \%)$, organizational - $3(10 \%)$, social - $5(16 \%)$, marketing-1 $(3 \%)$.

The summary table 1 is presented with the calculation of the correlation-regression analysis of organizational and managerial factors in connection with their influence on the activity in the exchange of knowledge.

Table 1. Results of regression analysis of organizational and managerial factors and individual activity of knowledge exchange

\begin{tabular}{|l|c|}
\hline \multicolumn{1}{|c|}{ Variable } & $\begin{array}{c}\text { Individual activity in the exchange of } \\
\text { knowledge }\end{array}$ \\
\hline Coordination mechanism "Formalization" & $0,265212394\left(\mathrm{R}^{2}=0,07\right)$
\end{tabular}


Table 1. Continued

\begin{tabular}{|l|c|}
\hline Coordination mechanism "Direct control" & $0,436623317\left(\mathrm{R}^{2}=0,2\right)$ \\
\hline Coordination mechanism "Mutual agreement" & $0,649091781\left(\mathrm{R}^{2}=0,8\right)$ \\
\hline The functional role of the leader & $0,70544556\left(\mathrm{R}^{2}=0,49\right)$ \\
\hline Team (emotional) role of the leader & $0,543776014\left(\mathrm{R}^{2}=0,3\right)$ \\
\hline Organizational environment & $0,576853167\left(\mathrm{R}^{2}=0,33\right)$ \\
\hline Organizational conditions for knowledge exchange & $0,62298654\left(\mathrm{R}^{2}=0,38\right)$ \\
\hline Knowledge exchange reward system & $0,62\left(\mathrm{R}^{2}=0,38\right)$ \\
\hline
\end{tabular}

Also, during the pilot study, verbal formulations were additionally tested and corrected, possible difficulties with filling out the questionnaire were identified and eliminated. Additionally added filtering questions.

\section{Conclusions}

The use of cross-functional teams allows companies to respond in a timely, flexible and adequate manner to changes in the external business environment and ensure the exchange of information and knowledge within the entire organization, thereby increasing the potential for competitiveness and ensuring its sustainable development. Also, the activity in the conditions of cross-functional teams allows to comprehensively and simultaneously work out various functional aspects of the project, thereby increasing the productivity and efficiency of the team, which means that cross-functional teams can be considered as highly productive work practices.

Cross-functional team management is considered as a part of the overall management and HR management of the company. The greatest challenge in the work of crossfunctional teams is to establish communication and effective knowledge exchange between its participants.

To overcome barriers to knowledge exchange, it is necessary to use such organizational and managerial solutions that will promote the internal interest of team members in knowledge exchange, establish interpersonal relations and effective communication channels.

\section{References}

1. J. Storey, Human Resource Management: A Critical Text. $3^{\text {rd }}$ ed. (2007)

2. J. Pfeffer, The formula for business success: people come first (2006)

3. M. Huselid, Russian Management Journal, 6(3), 87 (2008)

4. G. Tett, The Silo Effect: The Peril of Expertise and the Promise of Breaking Down Barriers (2015)

5. R. Santa, M. Ferrer, P. Bretherton, P. Hyland, Team Performance Management, 16 (3), 148 (2010)

6. E.P. Kostenko, Journal of economic regulation, 9 (4), 107 (2018)

7. R.A. Proehl, Leadership \& Organization Development Journal, 17 (5), 3 (1996)

8. C.E Armstrong, C.A. Lengnick-Hall, Journal of Strategy and Management, 6 (1), 4 (2013)

9. S. Ghobadi, J. D'Ambra, Journal of Knowledge Management, 16 (2), 285 (2012) 